\title{
Direct continuous electromyographic control of a powered prosthetic ankle for improved postural control after guided physical training: A case study
}

\author{
Aaron Fleming ${ }^{1} \mathbb{D}$, Stephanie Huang ${ }^{1}$, Elizabeth Buxton ${ }^{2}$, Frank Hodges ${ }^{3}$ and He Helen Huang ${ }^{1, *}$ \\ ${ }^{1}$ Joint Department of Biomedical Engineering, North Carolina State University and University of North Carolina at Chapel Hill, \\ Raleigh, North Carolina 27606, USA \\ ${ }^{2}$ UNC Hospitals, Department of Rehabilitation Therapies, Chapel Hill, North Carolina 27514, USA \\ ${ }^{3}$ Prosthetic and Orthotic Fabrication, SunStone Lab LLC, Raleigh, North Carolina 27615, USA \\ *Corresponding author: Email: hhuang11@ncsu.com
}

Received: 03 November 2020; Revised: 18 February 2021; Accepted: 20 February 2021

Key words: dynamic standing balance; myoelectric control; postural control; training; transtibial amputee

\begin{abstract}
Despite the promise of powered lower limb prostheses, existing controllers do not assist many daily activities that require continuous control of prosthetic joints according to human states and environments. The objective of this case study was to investigate the feasibility of direct, continuous electromyographic (dEMG) control of a powered ankle prosthesis, combined with physical therapist-guided training, for improved standing postural control in an individual with transtibial amputation. Specifically, EMG signals of the residual antagonistic muscles (i.e. lateral gastrocnemius and tibialis anterior) were used to proportionally drive pneumatical artificial muscles to move a prosthetic ankle. Clinical-based activities were used in the training and evaluation protocol of the control paradigm. We quantified the EMG signals in the bilateral shank muscles as well as measures of postural control and stability. Compared to the participant's daily passive prosthesis, the dEMG-controlled ankle, combined with the training, yielded improved clinical balance scores and reduced compensation from intact joints. Cross-correlation coefficient of bilateral center of pressure excursions, a metric for quantifying standing postural control, increased to $.83( \pm .07)$ when using dEMG ankle control (passive device: $39( \pm .29)$ ). We observed synchronized activation of homologous muscles, rapid improvement in performance on the first day of the training for load transfer tasks, and further improvement in performance across training days $(p=.006)$. This case study showed the feasibility of this dEMG control paradigm of a powered prosthetic ankle to assist postural control. This study lays the foundation for future study to extend these results through the inclusion of more participants and activities.
\end{abstract}

\section{Introduction}

Recent advances in intelligent, powered prosthetic legs have opened up opportunities for individuals with lower limb amputations to restore their normative movements in a variety of walking contexts (Hitt et al., 2007; Au et al., 2008; Sup et al., 2009; Rouse et al., 2013; Liu et al., 2014; Tucker et al., 2017; Lenzi et al., 2018; Quintero et al., 2018; Wen et al., 2019). These modern devices primarily use autonomous control, which has not been demonstrated to assist other important daily tasks that involve unpredictable, noncyclic motor behavior and require continuous coordination with the user's motor control and 
environments. One example of such activities is anticipatory and compensatory postural control in standing, walking, or other recreational activities (Legro et al., 2001; Brandt et al., 2017).

Focusing on standing postural control, lower limb amputees wearing passive prostheses have shown decreased postural stability and increased compensation from the intact limb (Bolger et al., 2014; Ku et al., 2014). This is partly because of the lack of active degrees of freedom in the prostheses. Powered prostheses have active, controllable joints and, therefore, a potential to enhance the amputees' postural stability. Unfortunately, there has been no autonomous control solutions to assist amputees' standing posture yet because it is difficult to predict the postural perturbations and human anticipatory and compensatory control for counteracting the perturbations. We are aware of one research group, developing autonomous prosthesis control to assist posture stability of the prosthesis users when standing on slopes (Lawson et al., 2011). This automated control was reactive and limited in function because it can assist standing posture on a slope only, and it acted only after the prosthesis foot was on an incline. Hence, this prosthesis control was insufficient to assist anticipatory postural control (i.e., action before the perturbation happens) or handle the postural control under dynamic perturbations (e.g., weight transfer), which requires continuous postural control based on the shift of center of mass.

Since the human neural control system is highly adaptable to the task context, perhaps neural control of prosthetic joints can be a viable solution to assist the amputee's postural control and balance stability. EMG signals of the residual muscles are readily available efferent neural sources in amputees and have been used for neural control of prosthetic legs in walking. Many groups have used EMG pattern recognition to classify the user's locomotor tasks, switching autonomous prosthesis control mode accordingly for enabling seamless locomotor task transitions (Hargrove et al., 2009; Huang et al., 2009; Huang et al., 2011; Miller et al., 2013; Zhang and Huang, 2013). Another group used EMG signal magnitude recorded from the residual medial gastrocnemius (GAS) to proportionally modulate a control parameter in the automated prosthesis control during the push-off phase of walking (Wang et al., 2013). Both approaches relied on autonomous prosthesis control laws and do not produce neural control of prosthetic joints continuously. In human neuromusculoskeletal system, efferent neural signals activate muscles that then produce force continuously around a joint for limb movement. This inspired three other groups to design direct EMG (dEMG) control, in which EMG magnitude of one or a pair of residual antagonistic muscles are directly mapped to modulate the applied torque to the prosthetic joints continuously (Dawley et al., 2013; Huang et al., 2016; Clites et al., 2018). These groups conducted case studies to show the feasibility of dEMG on amputees in walking. Note that the existing studies on EMG control of powered prosthetic legs, regardless the methods used, focuses on locomotor tasks mainly. Little effort has been aimed to address postural control.

Based on the current body of work, however, it remains to be seen whether multi-input dEMG control of active prosthesis ankle is a feasible approach to assist noncyclic, dynamic postural control tasks like picking objects up from the ground (termed load transfer). One of the questions is whether the nervous system in human can still coordinate the recruitment of residual muscles that no longer have biomechanical function. Previous studies have shown a large variation among transtibial amputees in producing coordinated activity between the residual tibialis anterior (TA) and GAS in a sitting posture or walking (Clites et al., 2018; Huang and Huang, 2018; Huang and Huang, 2019). These results implied that individuals with transtibial amputations might no longer manifest normative activation in the residual muscles due to the limb amputation. Luckily, there has been evidence to show that training or practice is a potential way to improve the capability of amputees in modulating residual muscles' activity for dEMG control. Our previous study (Fleming et al., 2019) tested transtibial amputees in dEMG control of a virtual inverted pendulum, mimicking the dynamics of standing posture. We noted improved task performance for all the amputee participants after a short-term practice within the same experimental visit. However, the amount of improvement varied significantly among the participants. Acclimation to dEMG control has involved repeating the evaluated task (walking) for an extend period of time (Dawley et al., 2013; Huang et al., 2016), or visualizing phantom limb movements (Clites et al., 2018). For Huang et al. (2016), transtibial amputees did not adapt activation of their residual GAS until they were given visual feedback of their prosthetic ankle 
angle with a target trajectory. However, it was unclear whether, after removing biofeedback training, amputees could still reproduce desired ankle joint trajectories or continue to improve control. From the findings of previous studies, we postulate that amputees might adapt and learn the necessary muscle activation pattern for control function after training and practice.

In this study, we expand the work of previous studies by (a) designing a biomimetic dEMG control paradigm using residual TA and GAS muscles (antagonistic muscles) for an artificial muscledriven prosthesis ankle, (b) creating and implementing a 4-week physical therapist (PT)-guided training paradigm (without artificial feedback to the prosthesis user), and (c) investigating the ability for an amputee to improve standing postural control with this control paradigm. We aim to demonstrate the feasibility and potential benefit of a multi-input dEMG control paradigm of a powered ankle prosthesis, combined with PT-guided training, on an individual with a transtibial amputation for enhanced postural stability. The results of this case study may inform the design of dEMG control of motorized prosthetic ankle, training protocol associated with this control paradigm, and the future development of versatile powered prostheses that can assist various activities of individuals with transtibial amputations.

\section{Materials and Methods}

\section{Participant}

We recruited one amputee participant to take part in this case study. The participant provided informed, written consent to participate in this Institutional Review Board approved study at the University of North Carolina at Chapel Hill. The participant was 57 years old and 3 years post-amputation with septic shock as the cause. The participant weighed $131 \mathrm{~kg}$. The participant used a pin-lock suspension and a Pro-Flex foot (Össur) daily. For the purpose of the study, the participant was fit with a new prosthetic socket (StabileFlex, Coyote Design). This transtibial socket design provided more room in the anterior-posterior direction while still maintaining adequate fit by loading the medio-lateral sides of the residual limb more heavily. This socket design provided more room for the residual TA and residual GAS muscles to contract compared to traditional socket designs, which increased comfort of residual muscle contractions within the socket and reduced residual muscle fatigue. On a daily basis, the participant used his passive prosthesis for household and community ambulation. He was able to traverse environmental barriers without requiring an assistive device and was independent with daily tasks, including driving. In a previous study, this participant demonstrated relatively average task performance compared with other amputee participants when controlling a continuous, dynamic virtual task with residual antagonistic shank muscles (Fleming et al., 2019) (participant TT2).

\section{Clinical Screening}

We conducted a sensory screening of the participant before the start of the study. A trained physical therapist performed a sensation screen of the participant's residual and intact limb. We noted partial neuropathy in the participant's intact foot. The participant had diminished light touch sensation distal to the ankle joint. The participant had absent light touch sensation at the medical aspect of the intact foot. Above the ankle joint, the participant was able to localize light touch sensation stimuli in all dermatomes bilaterally.

\section{Device Design and Control}

To mimic the movement production in biological joints, we propose a dEMG control of an experimental ankle prosthesis driven by pneumatic artificial muscles (PAMs) (Huang et al., 2014). PAMs are rubber, linear actuators with contraction dynamics and length dependent force generation relationships similar to normative musculature (Huang et al., 2014). Two PAMs in front of the socket replace the function of TA; and two PAMs on the opposite side functions as the GAS. The force production of TA- and 
GAS-mimicking PAMs (like biological muscles) is length-dependent and modulated by the EMG magnitude recorded from residual TA and GAS, respectively.

$$
\begin{gathered}
F_{i}=u_{i}\left(b * l_{i}+f_{0}\right), \\
u_{i}=.1 *\left(u_{\text {active }, i}+u_{\text {baseline }, i}\right),
\end{gathered}
$$

where $b[N / m]$ is the slope of force production, $F_{i}[N]$, where $I=1,2$ for the GAS and TA, respectively. $f_{0}[N]$ is the offset and the functional lengths $l_{1}$ and $l_{2}$ of the PAMs were .240-.275 m. The input control signal $[V]$ was comprised of baseline signal $u_{\text {baseline, } 1,2}$ used to generate the set stiffness of the prosthetic ankle and $u_{\text {active, } 1,2}$ was the active control signal from TA and GAS residual muscle activity, respectively. In this study, we implemented continuous control of both dorsi- and plantar-flexion using two sets of proportional pressure valves (MAC Valves, Wixom, MI) with two valves allocated to each PAM for a total of eight valves. The input control signal for the control valves was $0-10 \mathrm{~V}$ which corresponded to a pressure output of 0-90 psi proportionally. These control signals are empirically multiplied by 1 so that $u_{i}$ is expressed as a unit vector $(0-1 \mathrm{~V})$. Our prosthesis prototype used the same setup reported previously (Huang et al., 2014); the dynamics of the PAMs and the device can also be found in this previous report.

We processed EMG signals from residual TA and residual lateral GAS muscle in real-time (dSPACE, CLP-1103, 0-10 V output) to create a smoothed control signal for each set of pressure valves (Figure 1). The real-time setup created a smoothed control signal by first applying a high-pass filter $(100 \mathrm{~Hz}$, second order Butterworth) to reduce the effect of potential signal artifacts. The setup then rectified the signal and applied a low-pass filter ( $2 \mathrm{~Hz}$, second order Butterworth). The smoothed signal for each respective muscle was then sent to the pressure regulators, which generated pressure proportionally to the input voltage to actuate the device (Figure 1).

We applied a baseline signal $\left(u_{\text {baseline, } 1,2}\right)$ from the setup for both pairs of muscles to set a base stiffness for the ankle prosthesis. While the dEMG control was off, and the prosthesis unloaded, we applied a baseline signal that generated a neutral ankle position $\left(5-7^{\circ}\right.$ dorsiflexion). Then, we then asked the participant to stand and asked him to compare the stiffness of the pneumatic device with his intact side while shifting his weight. We adjusted baseline signals based on the perceived baseline stiffness to more closely match his perceived intact ankle stiffness. We then repeated this process until both neutral ankle angle and perceived stiffness met both criteria. Through this process we established a baseline signal of $\sim 3 \mathrm{~V}$ for the plantar- and dorsi-flexor muscles. When the participant had active control (dEMG control
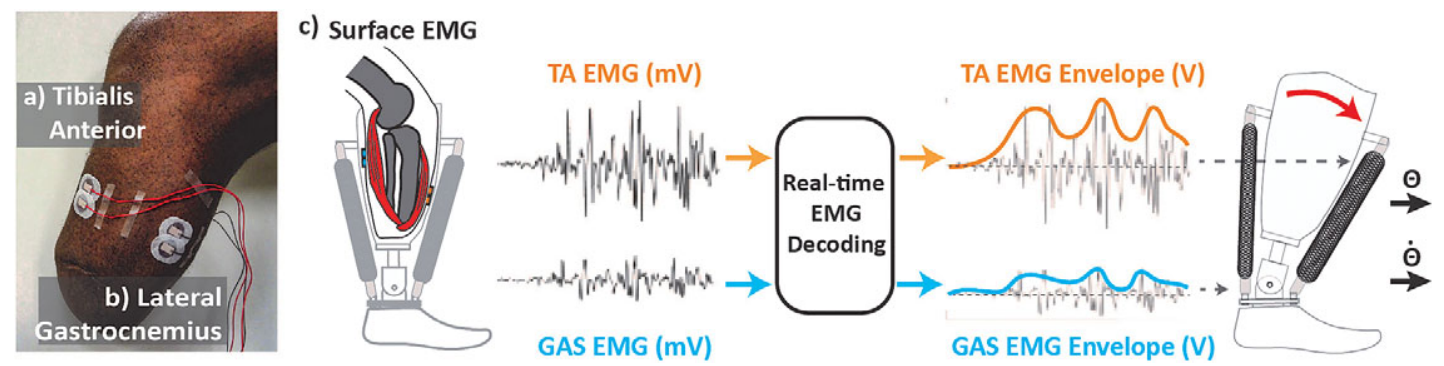

Figure 1. Real-time control setup. (a) Tibialis anterior electrode placement. (b) Lateral gastrocnemius electrodes placement. Electrodes are placed in line with muscle bellies (location determined through palpation as amputee is asked to contract muscle). Cables are routed away from bony landmarks. (c) Real-time electromyographic (EMG) processing. EMG activity is collected and processed to generate smooth control signal proportionally modulating the magnitude of air pressure within pneumatic artificial muscles (PAMs). Contractile force from PAM generates change torque and stiffness at prosthetic ankle joint. 
was turned on) we observed an average tonic activity from the residual muscles $(\sim 1.3 \mathrm{~V}$ from residual GAS, $\sim 1 \mathrm{~V}$ from residual TA) across sessions. In order to allow the participant true continuous control of the prosthetic device, we did not enforce an EMG threshold that would restrict low-level activity from controlling the device. We applied a gain to each control signal at the beginning of each session in order that a maximum contraction generated a control signal between 9 and $10 \mathrm{~V}$.

\section{Experimental Protocol}

Before the initial evaluation and training, we introduced the amputee participant to the direct EMG control paradigm and the pneumatic ankle device. While sitting, the participant wore the powered ankle prosthesis and was given time to freely move the ankle joint via residual muscle contractions. During this free exploration, we provided visual feedback of his residual muscle activation as a percentage of his maximum voluntary contraction. In order to facilitate learning the dynamics (i.e., possible combinations of ankle joint stiffness) we then asked the participant to fill a virtual control input space with his residual antagonistic muscle contractions (as described in Huang and Huang, 2019). We then repeated these steps while the amputee participant stood with handlebar support available to him. We took these steps to provide the participant with a clear understanding of the input-output relationship of reciprocal activation and co-activation of his residual muscles to changes prosthetic ankle joint dynamics. After this introduction stage we did not provide the amputee participant visual feedback of residual muscle activations.

The study consisted of an initial evaluation, five training sessions, a final evaluation, and a supplementary evaluation. The timeline for training and evaluation sessions are outlined (Table 1).

For the evaluation sessions, we asked the participant to perform quiet standing tasks across various sensory conditions. The four tasks selected involve quiet standing under two visual conditions, Eyes Open (EO) and Eyes Closed (EC), and two surface conditions, Firm and Foam, as described by the BESTest (Horak et al., 2009). These tasks were scored by a trained physical therapist on a scale from 0 to 3 where when the participant stood stably for $30 \mathrm{~s}($ Score $=3), 30 \mathrm{~s}$ unstable (Score $=2)$, stood less than $30 \mathrm{~s}$ $($ Score $=1)$, and unable (Score $=0)$ (Horak et al., 2009).

For the training sessions, we selected tasks relevant to daily life activities: load transfer, sit-to-stand, forward reach, and arm raise. These tasks (with the exception of the load transfer) are also a subset of evaluation tasks in the BESTest (Horak et al., 2009). We selected these training tasks to differ from the evaluation tasks in order to understand the effect of training to overall standing stability, as opposed to task-specific stability, while using the dEMG control of a prosthetic ankle. At the start of each training session, we asked the amputee to stand with his prosthetic foot on a rocker-board and intact foot on firm ground for $30 \mathrm{~s}$. During training, the participant completed two trials of each task per session with a minimum of four repetitions per trial. The number of repetitions increased across days, as prescribed by the physical therapist, where Day 4 of the training sessions (Table 1) had 20 total repetitions of each task.

Table 1. Clinical standing balance evaluation and training timeline

\begin{tabular}{|c|c|c|c|c|c|}
\hline Day & Day 1 & Day 3 & Day 5 Day 6 & Day 7 & Day 8 \\
\hline Session type & $\begin{array}{l}\text { Passive prosthesis and } \\
\text { dEMG prosthesis } \\
\text { evaluation }^{\mathrm{a}}\end{array}$ & Training (dEMG only) & & dEMG evaluation & $\begin{array}{l}\text { Supplementary } \\
\text { evaluation } \\
\text { (passive only) }\end{array}$ \\
\hline Tasks & $\begin{array}{l}\text { Quiet standing: } \\
\text { 1) Firm, EO } \\
\text { 2) Firm, EC } \\
\text { 3) Foam, EO } \\
\text { 4) Foam, EC }\end{array}$ & $\begin{array}{l}\text { Rocker board warm-up } \\
\text { Arm raise } \\
\text { Forward reach } \\
\text { Load transfer } \\
\text { Sit-to-stand }\end{array}$ & & $\begin{array}{l}\text { Quiet Standing: } \\
\text { 1) Firm, EO } \\
\text { 2) Firm, EC } \\
\text { 3) Foam, EO } \\
\text { 4) Foam, EC }\end{array}$ & $\begin{array}{l}\text { Rocker board } \\
\text { warm-up } \\
\text { Arm raise } \\
\text { Forward reach } \\
\text { Load transfer } \\
\text { Sit-to-stand }\end{array}$ \\
\hline
\end{tabular}


We conducted the study over the course of 25 days. We gave a minimum of 1 day of rest between sessions to reduce fatigue effects and a maximum of 4 days of rest between sessions to minimize learning losses. We conducted training with the dEMG controlled device only. We evaluated standing stability with both passive and dEMG controlled devices on the first day. After training, we performed a follow-up evaluation with the dEMG control. In order to compare postural control strategies in training tasks across devices we conducted a supplementary evaluation session where the participant repeated the training tasks while wearing his passive device.

A trained clinician attended each training session with the participant. During each training session, the clinician observed the participant complete each task. Between repetitions, the clinician provided feedback to the participant regarding his full-body symmetry, body mechanics, foot positioning, and alignment. The clinician provided feedback to encourage equal contribution from both limbs toward the specific task. The patient received verbal cues to shift his weight onto his prosthetic side and to recruit muscles in a "toes up" or "toes down" direction when learning each task. This directional cue is the same language used when he performed his warm-up on the rocker board. He also required cues to shift his weight onto his prosthetic side, especially for tasks such as sit to stand transfers in which he was accustomed to compensating for an ankle that was relatively fixed, whereas the power prosthesis allowed for movement in the sagittal plane.

\section{Measurements}

During all sessions, we recorded activity from the residual and intact shank muscles. Specifically, we placed EMG sensors (Neuroline 715, $1 \mathrm{~mm}$ height, Ballerup, Denmark) on residual lateral GAS and residual TA muscles (Figure 1). We located residual muscle bellies via palpation while the participant contracted and relaxed his muscles (Huang and Ferris, 2012). We then routed cables away from bony landmarks and connected them to a preamplifier (Motion Lab Systems, MA-412, Gainx20, Baton Rouge, LA) outside of the prosthetic socket. We placed EMG sensors (Motion Lab Systems, MA-420, Gainx20) on intact GAS and intact TA muscles. We connected all sensors to an amplifier (MA300-XVI, Gain x1000).

For all sessions, we collected center of pressure $(\mathrm{CoP})$ locations under each foot using an instrumented split-belt treadmill $(1,000 \mathrm{~Hz}$, Bertec Corp., Columbus, OH). For the final session of training (Day 6) and the supplementary passive evaluation session (Day 8) we collected kinematics from the amputated limb using motion capture $(100 \mathrm{~Hz}, 15$ markers, VICON, Oxford, UK). In order to limit the setup duration in the training session we unfortunately did not capture full body kinematics and EMG signals in all the sessions.

\section{Data Analysis}

We processed all data offline using Matlab (Mathworks, Natick, MA). We analyzed all quiet standing trials where the participant was able to maintain balance for the entire trial without stepping. Since the participant was unable to maintain balance in the dEMG control, pre-training, foam condition, we used the score given by the physical therapist for comparison. For the training sessions and supplementary evaluation, we analyzed data from the load transfer tasks only. We selected the load transfer task for analysis since this was self-reportedly the most difficult task for the participant during training.

For the training session analysis, we extracted and evaluated each repetition of the load transfer task. Each repetition was manually extracted through visual inspection of the summed vertical ground reaction forces in order to determine the moment the weight was picked up (before pick-up the weight was located beside the instrumented treadmill). Based on the speed of movement during training, we empirically windowed each repetition to $\pm 2 \mathrm{~s}$ on either side of the moment of pick-up.

For all evaluation trials and load transfer repetitions, we calculated synchronization of CoP excursions in the Anterior-Posterior direction under each foot by taking the cross-correlation between the time series (Mansfield et al., 2011). For each trial, we subtracted the mean CoP values from each foot and conducted a cross-correlation of the times series using MATLAB (xcorr). We determined the cross-correlation coefficient at time zero $\left(\mathrm{CC}_{0}\right)$, max cross-correlation coefficient $\left(\mathrm{CC}_{\max }\right)$, and the lag value $\left(\mathrm{Lag}_{\mathrm{CC}}\right)$ in 
milliseconds from time zero to $\mathrm{CC}_{\max } \cdot \mathrm{CC}_{\max }$ and $\mathrm{Lag}_{\mathrm{CC}}$ are calculated to determine potential lag in $\mathrm{CoP}$ excursions between limbs using a window of \pm 1 s (Mansfield et al., 2011).

For the final training session (dEMG control) and in the supplementary session (passive), we analyzed ankle, knee, and hip joint flexion during the load transfer task. We calculated joint angles in the sagittal plane (Dionisio et al., 2008) for each windowed repetition. We then subtracted joint angles during quiet standing from all repetitions for each condition. We tabulated peak hip, knee, and ankle flexion angles during the windowed repetitions.

In order to analyze the neural control strategy used by the participant, we processed EMG activity from residual and intact TA and GAS muscles. First, we high-pass filtered the raw EMG signal (Butterworth, second order, $100-\mathrm{Hz}$ cutoff) from all muscles to remove potential motion artifacts. We rectified the signals and applied a low-pass filter (Butterworth, second order, 20-Hz cutoff) in order to generate a smoothed signal for qualitative comparison. We then selected representative repetitions from the first and final day of training based on $\mathrm{CC}_{0}$ values that were closest to the average $\mathrm{CC}_{0}$ for that day of training. We then plotted CoP excursion, EMG activity from residual and intact TA and GAS, and residual TA and GAS control signals together for qualitative comparison.

\section{Statistical Analysis}

For our statistical analysis of the data, we used the statistical software (JMP, SAS, Cary, NC). We used a one-way ANOVA to compare the $\mathrm{CC}_{0}, \mathrm{CC}_{\max }$, and $\mathrm{Lag}_{\mathrm{CC}}$ with training day as the main effect. We used the Shapiro-Wilk normality test $(p<.01)$ to detect outlier repetitions. One repetition was removed from our analysis (Repetition 2, Day 1 training, $\mathrm{CC}_{0}=-.4$ ). We ran a simple linear regression to determine the amount of variance (via $R$-squared) described by trial order in each training session $\mathrm{CC}_{0}, \mathrm{CC}_{\max }$, and $\mathrm{Lag}_{\mathrm{CC}}$. We analyzed joint flexion angles in the load transfer task between dEMG control and passive device. We used a two-way ANOVA to detect main and interaction effects of Device and Joint. When we found a significant effect, we tested for statistical differences within joint and device conditions using Tukey's honestly significant difference test $(a=.05)$. We set significance threshold using an alpha value of .05 .

\section{Results}

\section{Quiet Standing Evaluation: Clinical Scoring of Stability}

We observed clear improvements in stability with the dEMG control of the powered ankle in the quiet standing tasks post-training (Table 2). In the pre-training condition, the amputee displayed moderate instability on the firm surface for both eyes open and eyes closed, evidenced by visually noticeable sways $($ Score $=2)$. In the foam surface, the amputee was unable to maintain balance without stepping in either

Table 2. Quiet standing tasks clinical score and between limb synchronization

\begin{tabular}{lllcc}
\hline Device & Surface & Condition & Score (BESTest) & $\mathrm{CC}_{0}$ \\
\hline Passive & Firm & Eyes Open & 2 & .654 \\
& \multirow{2}{*}{ Foam } & Eyes Closed & 2 & .395 \\
& & Eyes Open & 2 & .540 \\
dEMG control (pre-training) & \multirow{2}{*}{ Firm } & Eyes Closed & 2 & -.004 \\
& \multirow{2}{*}{ Foam } & Eyes Open & 2 & .460 \\
& \multirow{2}{*}{ Firm } & Eyes Open & 2 & .590 \\
\multirow{2}{*}{ dEMG control (post-training) } & Eyes Closed & 1 & Insufficient data (9 s max) \\
& \multirow{2}{*}{ Foam } & Eyes Open & 3 & Insufficient data (2 s max) \\
& & Eyes Open & 3 & .852 \\
& & Eyes Closed & 3 & .862 \\
& & & .713 \\
\hline
\end{tabular}


condition $($ Score $=1)$. Post-training, the amputee improved stability over all conditions $($ Score $=3)$. In all surface and vision conditions the participant did not display visually significant sways and did not require the use of any handlebars.

We observed differences in stability between the passive (baseline) and dEMG controlled condition (Post-training) (Table 2). With his passive device the amputee was able to maintain balance in all conditions with significant postural sway, and the use of handlebars was not needed (Score $=2$ ). With dEMG control, post-training, the amputee had minimal postural sways for all conditions $($ Score $=3$ ).

\section{Quiet Standing Evaluation: Between Limb Synchronization}

The participant demonstrated distinct patterns of bilateral center of pressure excursions between the passive and $\mathrm{dEMG}$ control (Post-training) for the quiet standing tasks. Figure 2 shows this stark contrast in the foam condition where the participant displayed noticeably higher synchronization between his intact and prosthetic foot $\mathrm{CoP}_{\mathrm{AP}}$ excursion with dEMG control $\left(\mathrm{EO} \mathrm{CC}_{0}=.713, \mathrm{EC} \mathrm{CC}_{0}=.867\right)$ compared with his passive device $\left(\mathrm{EOCC}_{0}=.540, \mathrm{EC} \mathrm{CC}_{0}=-.004\right)$. We observed this increase in synchronization during dEMG control in firm surface conditions as well (Table 1). The magnitude of $\mathrm{CoP}_{\mathrm{AP}}$ excursion of the prosthetic foot in the passive device was less than the intact limb $\mathrm{CoP}_{\mathrm{AP}}$ excursion as evidenced by time series plots (Figure $2 \mathrm{a}, \mathrm{b}$ ). The participant increased $\mathrm{CoP}_{\mathrm{AP}}$ excursion on the prosthetic side post-training with dEMG control (Figure 2c,d).

In dEMG control, the amputee demonstrated improvements in between limb synchronization after training for all quiet standing conditions (Table 2 and Figure 3). In the firm condition, pre-training, we observed moderate cross-correlation in $\mathrm{CoP}$ excursions between the intact and dEMG controlled foot $\left(\mathrm{EO} \mathrm{CC}_{0}=.460, \mathrm{EC} \mathrm{CC}_{0}=.590\right)$ (Figure 3a,b). Post-training, the participant more closely synchronized $\mathrm{CoP}$ excursions between the two feet $\left(\mathrm{EO} \mathrm{CC}_{0}=.852, \mathrm{EC} \mathrm{CC}_{0}=.862\right)$ (Figure $\left.3 \mathrm{c}, \mathrm{d}\right)$ in the firm condition. During the initial evaluation the amputee was unable to maintain balance in the foam condition thus we did evaluate $\mathrm{CC}_{0}$ for the pre-training, dEMG control condition. However, the amputee demonstrated similar synchronization values between firm and foam conditions in the post-training condition (Foam: $\left.\mathrm{EO} \mathrm{CC}_{0}=.713, \mathrm{EC} \mathrm{CC}_{0}=.867\right)$.

\section{Training Evaluation: Load Transfer Task}

Over the course of training, the amputee significantly improved between-limb synchronization of CoP excursion. In the initial trials of the load transfer task, the participant displayed moderate levels of synchronization $\left(\mathrm{CC}_{0}=.49( \pm .16), \mathrm{CC}_{\max }=.52( \pm .14), \mathrm{CC}_{\text {lag }}=-107.3 \mathrm{~ms}( \pm 357.1)\right)$ (Figure 4) similar to synchronization values observed during the initial evaluation. We observed that $\mathrm{CC}_{\max }$ and $\mathrm{CC}_{0}$ improved significantly over the course of just the first day, where $\mathrm{CC}_{\max }$ and $\mathrm{CC}_{0}$ are significantly related to repetition order $\left(\mathrm{CC}_{\max }: R^{2}=.459, p=.045 ; \mathrm{CC}_{0}: R^{2}=.646, p=.009\right)$ (Figure 4). We determined this relationship was significant for the first day, however, not for the trial order in the remaining days. Across training, day was found to be a significant main effect for $\mathrm{CC}_{\max }(p=.011)$ and $\mathrm{CC}_{0}(p=.006)$, but not for $\mathrm{CC}_{\text {lag }}(p=.279)$. At the final day of training, we observed $\mathrm{CC}$ values of $\left(\mathrm{CC}_{0}=.76( \pm .15), \mathrm{CC}_{\max }=.76\right.$ $\left.( \pm .16), \mathrm{CC}_{\text {lag }}=-22.8 \mathrm{~ms}( \pm 32.79)\right)$.

Analysis of EMG patterns during representative load transfers demonstrated distinct neural strategies between initial and final trials (Figure 5). These specific repetitions were chosen since their $\mathrm{CC}_{0}$ value closely matched average $\mathrm{CC}_{0}$ values for the initial and final day of training. Pre-training, we observed different timing and shape of EMG activity between the residual and intact limb. The participant intermittently activated the intact TA (Figure 5a) with a steady contraction of the GAS muscle throughout the movement (Figure 5b). In comparison, the amputee had little to no activation from the residual TA before peak squat depth (Figure 5a,c) followed by significant activation of the GAS while returning to the standing posture (Figure $5 \mathrm{~b}, \mathrm{c})$. The control signal reached half of its force generating potential $(5 \mathrm{~V} \sim$ $50 \mathrm{psi}$ ) in the plantar-flexor direction during this movement (Figure 5c). Post-training, the strategy between the two limbs appeared more closely aligned. Activations from the residual TA were seemingly identical to activations from the intact TA (Figure 5e). Intact and residual GAS muscle activations were 

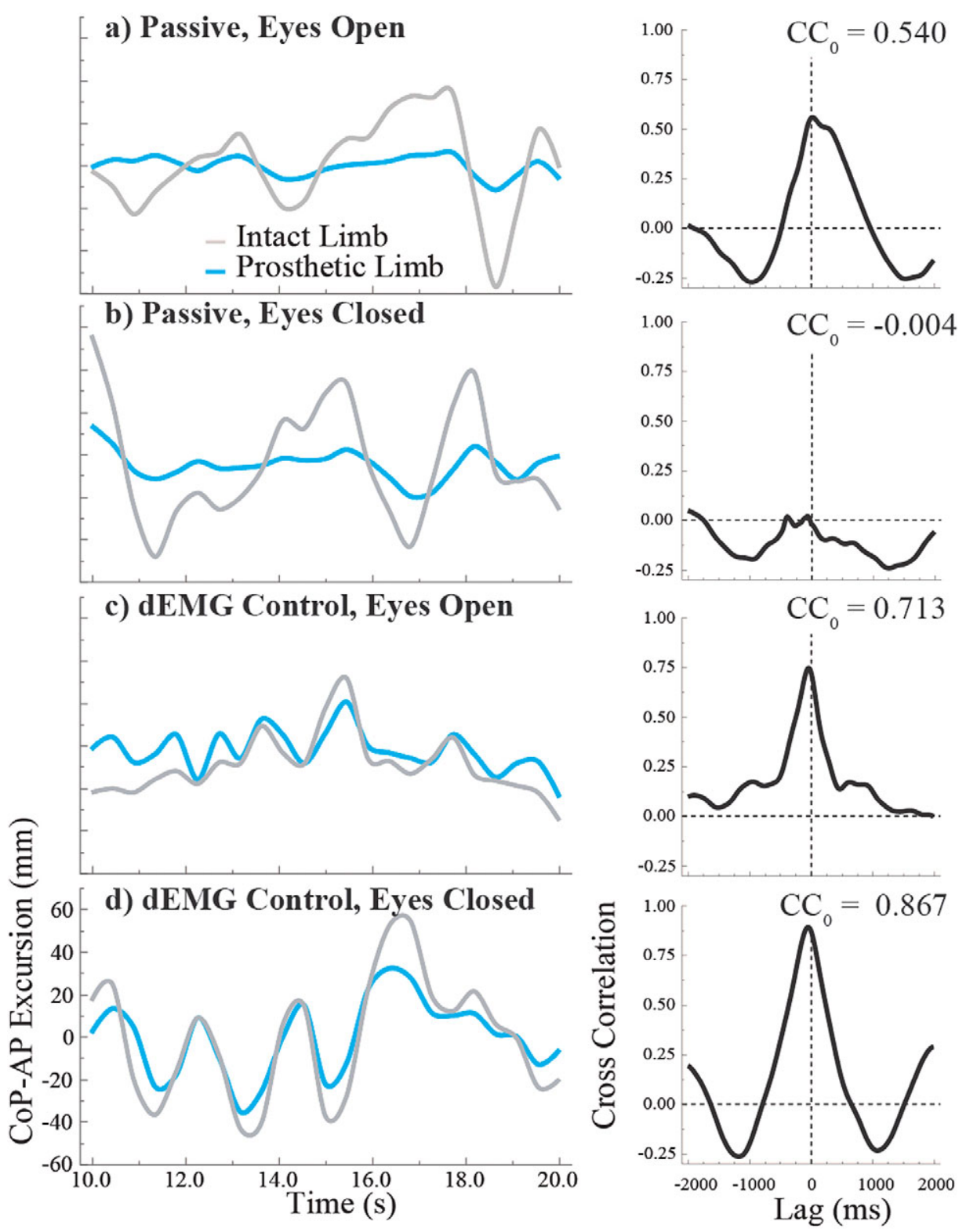

Figure 2. Passive versus post-training direct electromyographic (dEMG) control on the Foam Surface. Representative center of pressure excursion and cross correlation between limbs. Representative trials are 10 s portions taken from each 30-s trial. Trials shown above are foam surface only. (a) Passive device, eyes open condition. (b) Passive device, eyes closed condition. (c) dEMG controlled device, eyes open. (d) $d E M G$ controlled device, eyes closed.

relatively aligned (Figure $5 \mathrm{f}$ ) with the exception of activation of the intact GAS muscle before reversal of the squatting motion (Figure 5f). The control signal to the prosthesis (Figure 5c,g) mostly clearly demonstrated residual antagonistic pair control strategy across training. In final trials, we observed high activations of the residual TA at the beginning of the movement, followed by small contractions from the residual GAS and co-contraction post-squat (Figure 5g). $\mathrm{CC}$ of $\mathrm{CoP}_{\mathrm{AP}}$ excursions demonstrate the similarity in control strategy between limbs (Figure $5 \mathrm{~h}$ ). 


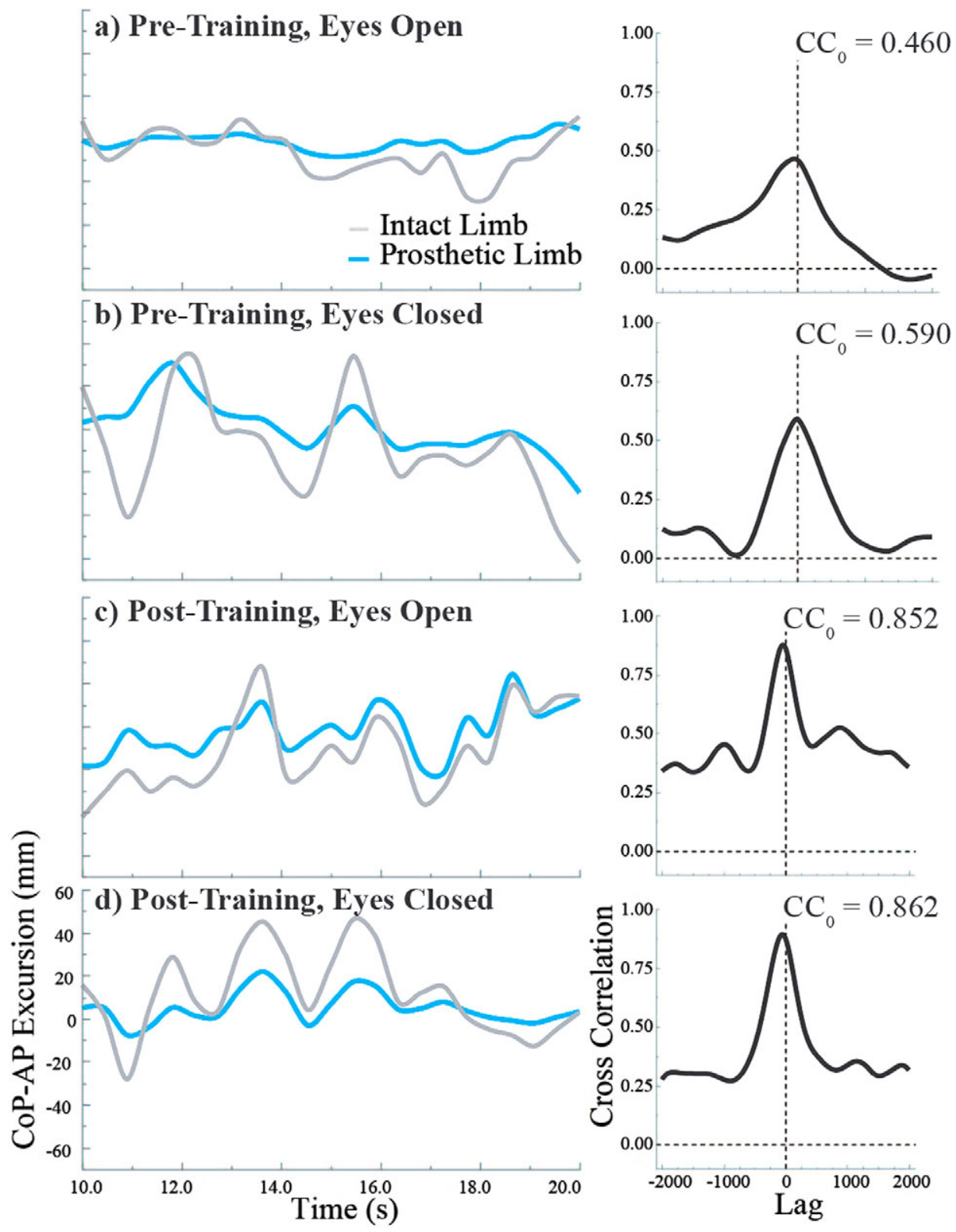

Figure 3. Pre-versus post-training with dEMG control on the firm ground. Representative center of pressure excursion and its cross correlation between limbs. Representative trials are 10 s portions taken from each 30-s trial. Trials shown above are firm surface only. (a) Pre-training, eyes open condition. (b) Pre-training, eyes closed condition. (c) Post-training, eyes closed condition. (d) post-training, eyes closed condition.

As a supplementary evaluation, we asked the participant to perform the load transfer task on the final evaluation day to determine the effect removing active EMG control with the pneumatic prosthesis. While the active control was switched off the participant conducted three repetitions of the load transfer task. For these trials, we observed $\mathrm{CoP}$ excursion $\mathrm{CC}_{0}$ for these repetitions to be $.465( \pm .14)$ ). 

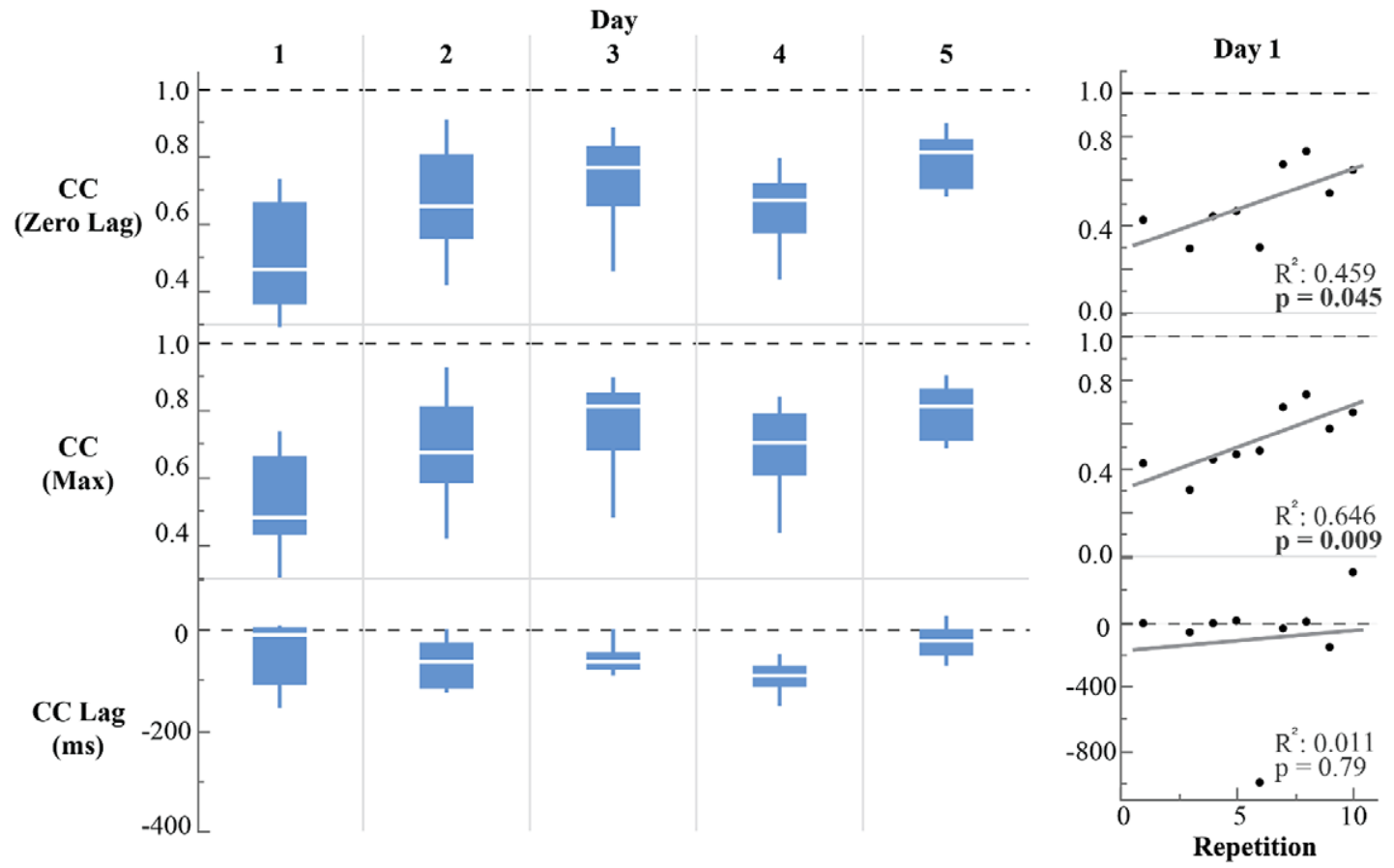

Figure 4. Center of pressure (CoP) synchronization values during training for the load transfer task. Rsquared values and $\mathrm{p}$-value are shown for cross-correlation $(C C)$ values (CC at zero lag, maximum CC, and lag of maximum CC from zero lag) for Day 1 of training. Due to concern for residual muscle fatigue during training, Days 1 and 2 contained less than 10 repetitions.

\section{Load Transfer Task: Postural Control Strategy}

Post-training, we observed significantly different postural strategies between the passive and dEMG controlled device for the load transfer task. We observed small flexion angles for the passive ankle prosthesis during the load transfer (Table 3 and Figure 6). With dEMG control post-training, the ankle flexion angle significantly increased (passive-dEMG, $p<.0001$ ). For the dEMG control condition the knee flexion angle also increased (passive-dEMG, $p<.0001$ ) and the hip flexion angle decreased (passive-dEMG, $p<.0001$ ). We observed a significant interaction between the device and joint $(p<.0001)$.

\section{Discussion}

In this study, we present the feasibility of direct EMG control to continuously operate prosthetic ankle joint mechanics to address the postural stability for individuals with transtibial amputation. The main finding of this study is that our recruited transtibial amputee participant was capable of using biomimetic, dual-input control of a PAM actuated prosthetic ankle to significantly improve standing postural control across various contexts compared with using a passive ankle prosthesis. Completely different from the "standard" control framework for active lower limb prostheses and exoskeletons as suggested in Tucker et al. (2015) that relies on preprogrammed, discrete finite state machines and prescribed control laws, dEMG control used in this case study, continuously drives a powered prosthesis joint based purely on the user's neural control signals (i.e. motor commands) from the residual GAS and residual TA muscles. This device offered the amputee user the freedom to continuously adjust the behavior of prosthetic ankle (i.e. control both stiffness and position via coactivity and reciprocal activity). The freedom to commit EMG-modulation of both stiffness and position allowed the amputee to more closely match interlimb 


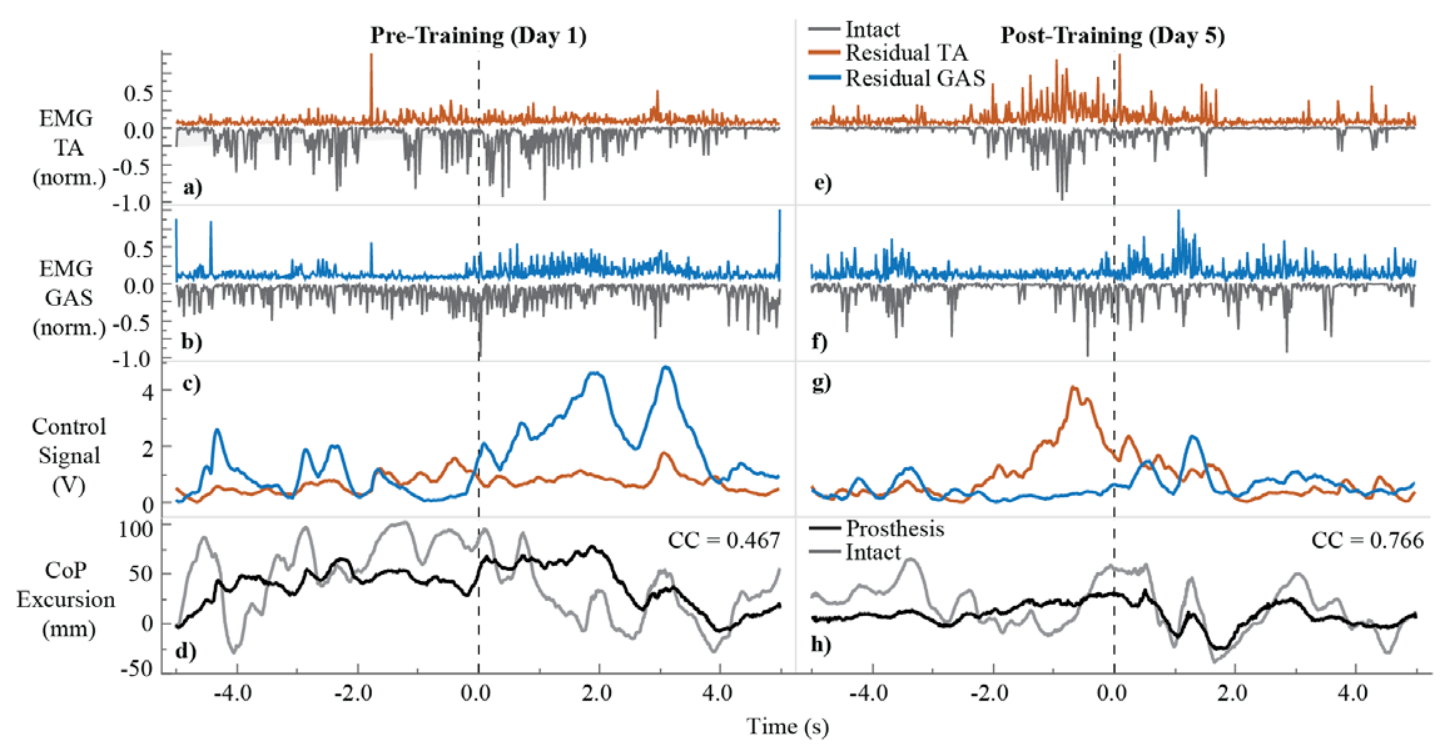

Figure 5. Representative load transfer trials pre- and post-training. Dashed line: moment of peak deceleration during squatting movement. (a) Normalized electromyography (EMG) of residual (orange) and intact (gray) tibialis anterior (TA) muscle pair. (b) Normalized EMG of residual (blue) and intact (gray) gastrocnemius $(G A S)$ muscle pair. (c) Control signal to the prosthesis from the real-time processing of residual TA (orange) and residual GAS (blue) muscle EMG. (d) Center of pressure (CoP) excursion from prosthetic (black) and intact foot (gray). Cross-correlation values are displayed for each representative trial (pre: cross-correlation $[C C]=.467$, post: $C C=.766)$. (e-h) Data for post-training. Normalized EMG was calculated by dividing the maximum EMG value for each muscle from the entire trial.

Table 3. Load transfer joint angle (passive vs. post-training dEMG control)

\begin{tabular}{|c|c|c|c|}
\hline \multirow[b]{2}{*}{ Joint } & \multicolumn{3}{|c|}{ Device } \\
\hline & Passive flexion (deg) & & dEMG control flexion (deg) \\
\hline Ankle & $4.07( \pm .71)$ & & $25.59( \pm 4.33)$ \\
\hline Knee & $51.00( \pm 3.51)$ & & $77.08( \pm 4.70)$ \\
\hline Hip & $103.89( \pm 5.07)$ & & $86.55( \pm 2.47)$ \\
\hline Device main effect & \multirow{3}{*}{\multicolumn{3}{|c|}{$\begin{array}{l}p<.0001 \\
p<.0001 \\
p<.0001\end{array}$}} \\
\hline Joint main effect & & & \\
\hline Interaction effect & & & \\
\hline
\end{tabular}

behavior across a range of tasks and conditions. We chose different postural control tasks during standing in this study, as the first step, to evaluate the potential of this biomimetic, dEMG control for standing postural control tasks that requires continuous coordination of residual muscle activation. This biomimetic control differs significantly from previous work since the amputee has active control of both plantar/ dorsiflexors with active and passive dynamic properties similar to normative musculature. Based on these results, future work should focus on implementing the properties of the proposed control into a motorized design that can be tested outside of the laboratory to assess its benefit to amputees' daily life.

One of the interesting observations in this study was that enabling neural control of a prosthetic ankle on the amputated side elicited improved motor coordination between the intact limb and amputated limb during postural control. The between-limb coordination was manifested by (a) synchronized CoP anterior-posterior excursion and (b) synchronized shank muscle activation. First, we observed a significant improvement in between-limb synchronization of $\mathrm{CoP}$ excursion during standing postural control 

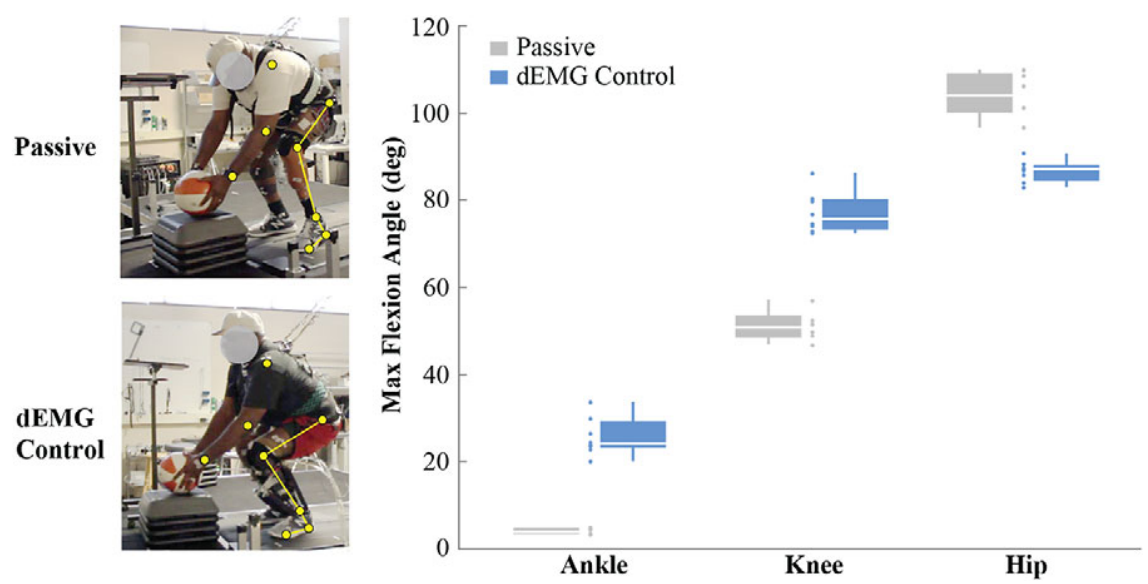

Figure 6. Load transfer task joint flexion angles (passive vs. post-training direct electromyographic [dEMG] control). Gray: passive prosthetic ankle, hip, and knee joint flexion on affected limb at peak squat depth (as determined by location of hip joint center). Blue: dEMG controlled prosthetic ankle, hip, and knee joint flexion at peak squat depth. Joint flexion angles determined as the difference between angle at maximum depth and angle during quiet standing.

when the TT amputee can actively use prosthetic ankle via neural control, compared to when he used passive device (Figures 2 and 3). Between-limb CoP synchronization has developed over recent years into a meaningful measure of postural control for populations with inter-limb deficits (i.e. stroke population) (Mansfield et al., 2011; Mansfield et al., 2012). When the participant wore a passive prosthesis, the missing ankle function led to lack of $\mathrm{CoP}$ excursion on the amputated side and therefore lack of bilateral CoP synchronization (Rusaw and Ramstrand, 2016). When the participant can actively move the ankle via the EMG control signals, not only the CoP excursion magnitude increased on the amputated side, but also it showed improved synchronization with the CoP excursion in the intact side. This CoP synchronization restores the possibility of normative CoP control strategies in standing typically observed in healthy individuals (i.e. CoP-CoM to CoM acceleration relationship Winter et al., 1998). The observation implies the importance in restoring ankle control and function for enhanced postural stability and the potential of dEMG control for active control of prosthetic ankle. Additionally, by demonstrating the ability for a transtibial amputee to volitionally adjust $\mathrm{CoP}$ excursion while improving standing postural control, this is the first study to show the potential for this biomechanical feature to indicate prosthetic ankle control capability. Second, the between-limb coordination was also observed in EMG activation pattern as shown in Figure 5. After learning the dEMG control of prosthetic ankle in standing postural control, nearly synchronized activation between intact and residual TA/GA was observed. One of the open questions is what neural mechanisms are responsible for the observed adaptation in residual muscle activations. The observation of synchronized activation in homologous muscles between limbs cause us to consider the potential for a common neural drive behind the activity for both muscles. It would be an interesting future direction to investigate the neuromuscular adaptation in lower limb amputees when the function of residual muscle activation is restored via $\mathrm{dEMG}$ control of prosthetic joints.

We attempted to evaluate whether, using this biomimetic control, the amputee could improve control over time without the use of artificial feedback of the prosthetic ankle state (i.e. visual feedback, nerve stimulation, etc.). Using resources already available in amputee rehabilitation (i.e. guided therapy with a physical therapist) we developed a specific, extended training paradigm guided by a physical therapist toward the training of dynamic, standing postural control tasks. Qualitatively, over the course of training, we witnessed various stages of learning from the amputee participant. During the initial training days ( 1 and 2) the amputee noted that he focused primarily on controlling the prosthetic ankle when completing the prescribed tasks. However, in the latter days of training (Days 3-5), the participant frequently 
mentioned focusing on whole-body movement, using his prosthetic and intact limb symmetrically. Huang et al. (2016) observed improvement in dEMG control of a prosthetic ankle during walking when they provided visual feedback of the ankle-joint angle, demonstrating the relevance of this joint-level focus when learning. We extend the results from this study by demonstrating the ability for an amputee to potentially continue the learning process beyond this joint level focus, without the use of visual feedback. Since this learning occurred in the absence of supplementary artificial feedback, only under the guidance of verbal feedback from a physical therapist, this type of training shows promise toward real-world application of dEMG control of a powered ankle prosthesis. While the stages of learning observed here are discussed qualitatively, future investigations of amputee learning the dEMG control of a prosthetic device would benefit by analyzing the potential change in multi-joint muscle coordination via muscle synergy analysis (Danna-dos-Santos et al., 2007; Latash, 2010).

Before conducting this study, we did not know whether our recruited amputee participant could coordinate his residual muscle activation appropriately for prosthetic ankle control to assist postural stability due to his limited capability in coordination of residual muscle activities. In addition, it was unclear how the participant's demographics, such as age (57 years old), body mass index ( 34$)$, presence of vascular disease (including partial neuropathy at the intact foot), might affect his ability to improve control during training. Although these factors may have a significant negative effect on standing postural control (Hageman et al., 1995; Lafond et al., 2004; Ku et al., 2012) they are highly characteristic traits of the lower-limb amputee population (Ziegler-Graham et al., 2008; Rosenberg et al., 2013). The beforetraining evaluation also showed limited muscle activation in residual TA (Figure 5) and comparable or even worse quiet standing test score (Table 2). In our previous study, we observed this amputee participant had relatively average residual muscle control when compared with other amputee participants (Fleming et al., 2019) (participant TT2). In the previous study, we asked amputee participants to coordinate antagonistic residual muscle activity to control a dynamic virtual inverted pendulum (similar to standing postural control). This participant demonstrated the capability of adapting residual muscle activity over time, however, it was unclear whether this adaptation could occur with a physical prosthetic device.

The results from this study have several implications for the potential clinical benefit of dEMG control of a powered prosthetic ankle. During the follow-up evaluation of the load transfer task, we observed the participant had limited range of motion with his passive prosthetic ankle, likely due to minimal change in angle of the stiff ankle joint. Hence, compensation with more trunk flexion was used, which is a known problem for back injuries during weightlifting. The participant was able to significantly change ankle angle using the dEMG control ankle allowing for an improved overall postural configuration (i.e., more vertical trunk angle) (Cole and Grimshaw, 2003) in lifting, which could significantly prevent secondary injuries post amputation. The implementation of neural control (as well as artificial sensory feedback) with powered prostheses has shown benefit toward prosthetic embodiment in the upper limb (Page et al., 2018). We expect the volitional, biomimetic control proposed in this current study to have a potential effect on prosthesis embodiment. There is truly a rich opportunity for the normalization of other functional tasks (like dancing, jumping, and picking up a child) that are critical to daily life activities and amputee quality of life. This control paradigm shows significant promise toward the restoration of these daily-life tasks. It is clear from these results that this control paradigm not only improved standing postural control for trained tasks, but also in untrained tasks (like standing on foam surface, Figure 2). The benefit of this direct control paradigm across multiple tasks poses significant benefits over automated control, whose algorithms must be developed for each individual tasks separately.

Our study included one amputee to investigate the feasibility of dEMG control of a powered ankle for enhanced postural control. Although exciting results were observed, this case study mainly served as a feasibility study and was insufficient to conclude the benefit of dEMG control of powered ankles on broader range of amputee population. Future work should expand this current case study to include more participants to understand the applicability to the general amputee population. It would be interesting in future study for more measures of stability (including center of mass, joint torque symmetry, etc.) to further inform the effect of dEMG control of a powered ankle prosthesis. While interlimb EMG activity more closely aligned post-training, it would be beneficial for future study to investigate joint torque to 
determine relative contribution of each joint toward task completion. Although its effect is not specifically addressed in the context of this study, future study would benefit to evaluate the effect of prosthetic socket design on residual muscle activations during EMG control of lower-limb prostheses. The reason for us to use PAM-driven prosthesis is that it is straightforward to formulate biomimicking ankle control. Ideally the same setup can be designed on motorized prostheses with virtual musculoskeletal model in the control software.

\section{Conclusion}

This case study was the first attempt to demonstrate the feasibility and potential for direct EMG control of a powered prosthetic ankle, combined with PT-guided training, to enhance standing postural control across various contexts and tasks. The participant when using dEMG-controlled powered ankle yielded improved clinical balance score, reduced compensation from the intact joints, and improved between-limb coordination, compared to those when using his daily passive prosthesis. In addition, the case study developed a PT-guided training protocol for transtibial amputees, which is necessary for them in learning dEMG control of powered ankle to assist postural control and improve postural stability. This case study has developed the grounds for future design of versatile and agile powered lower-limb prostheses via direct, continuous EMG control via residual muscles, which may further improve the motor function of individuals with lower limb amputations and improve the ability for amputees to navigate standing postural control tasks that are a significant portion of daily-life activities.

Acknowledgments. The authors would like to acknowledge Abby Sweitzer and Loryn Boorstein for their help with data collection. We would also like to thank all the undergraduate researchers and participants for their time and assistance with this research project.

Funding Statement. Funding for this research project was received from NIH NICHD F31HD101285, NIH EB024570, NSF1954587.

Competing Interests. The authors declare that they have no competing interests.

Authorship Contributions. S.H. and H.H. contributed to the design of the experiment. S.H., E.B., and F.H. conducted the experiments. A.F. and S.H. processed the data. A.F., S.H., and H.H. wrote the manuscript. All authors contributed to the analysis and interpretation of the results and read and approved the final manuscript.

Data Availability Statement. The datasets used and/or analyzed during the current study are available from the corresponding author on reasonable request.

Ethical Standards. The experimental protocol was approved by the Intuitional Review Board at the University of North CarolinaChapel Hill. All participants provided written, informed consent.

\section{References}

Au S, Berniker M and Herr H (2008) Powered ankle-foot prosthesis to assist level-ground and stair-descent gaits. Neural Networks 21, 654-666.

Bolger D, Ting LH and Sawers A (2014) Individuals with transtibial limb loss use interlimb force asymmetries to maintain multidirectional reactive balance control. Clinical Biomechanics 29, 1039-1047.

Brandt A, Wen Y, Liu M, Stallings J and Huang HH (2017) Interactions between transfemoral amputees and a powered knee prosthesis during load carriage. Scientific Reports 7, 14480.

Clites TR, Carty MJ, Ullauri JB, Carney ME, Mooney LM, Duval JF, Srinivasan SS and Herr HM (2018) Proprioception from a neurally controlled lower-extremity prosthesis. Science Translational Medicine 10, eaap8373.

Cole $\mathbf{M}$ and Grimshaw P (2003) Low back pain and lifting: a review of epidemiology and aetiology. Work 21, $173-184$.

Danna-dos-Santos A, Slomka K, Zatsiorsky VM and Latash ML (2007) Muscle modes and synergies during voluntary body sway. Experimental Brain Research 179, 533-550.

Dawley JA, Fite KB and Fulk GD (2013) EMG control of a bionic knee prosthesis: exploiting muscle co-contractions for improved locomotor function. IEEE Proceedings of the International Conference on Rehabilitation Robotics 2013, 6650389.

Dionisio VC, Almeida GL, Duarte M and Hirata RP (2008) Kinematic, kinetic and EMG patterns during downward squatting. Journal of Electromyography and Kinesiology 18, 134-143. 
Fleming A, Huang S and Huang H (2019) Proportional myoelectric control of a virtual inverted pendulum using residual antagonistic muscles: toward voluntary postural control. IEEE Transactions on Neural systems and Rehabilitation Engineering 27, 1473-1482.

Hageman PA, Leibowitz JM and Blanke D (1995) Age and gender effects on postural control measures. Archives of Physical Medicine 76, 961-965.

Hargrove LJ, Huang H, Schultz AE, B. Lock A, Lipschutz R and Kuiken TA (2009) Toward the development of a neural interface for lower limb prosthesis control. Annual International Conference of the IEEE Engineering in Medicine and Biology Society 2009, 2111-2114.

Hitt J, Bellman R, Holgate M, Sugar T and Hollander K(2007) The SPARKy (Spring Ankle with Regenerative Kinetics) Project: Design and Analysis of a Robotic Transtibial Prosthesis with Regenerative Kinetics. Las Vagas, NV, 1587-1596.

Horak FB, Wrisley DM and Frank J (2009) The balance evaluation systems test (BESTest) to differentiate balance deficits. Physical Therapy 89, 484-498.

Huang H, Kuiken TA and Lipschutz RD (2009) A strategy for identifying locomotion modes using surface electromyography. IEEE Transactions on Biomedical Engineering 56, 65-73.

Huang H, Zhang F, Hargrove LJ, Dou Z, Rogers DR and Englehart KB (2011) Continuous locomotion-mode identification for prosthetic legs based on neuromuscular-mechanical fusion. IEEE Transactions on Bio-Medical Engineering 58, $2867-2875$.

Huang S and Ferris DP (2012) Muscle activation patterns during walking from transtibial amputees recorded within the residual limb-prosthetic interface. Journal of Neuroengineering and Rehabilitation 9, 55.

Huang S and Huang H (2018) Voluntary control of residual antagonistic muscles in transtibial amputees: feedforward ballistic contractions and implications for direct neural control of powered lower limb prostheses. IEEE Transactions on Neural Systems and Rehabilitation Engineering 26, 894-903.

Huang S and Huang H (2019) Voluntary control of residual antagonistic muscles in transtibial amputees: reciprocal activation, coactivation, and implications for direct neural control of powered lower limb prostheses. IEEE Transactions on Neural Systems and Rehabilitation Engineering 27, 85-95.

Huang S, Wensman JP and Ferris DP (2014) An experimental powered lower limb prosthesis using proportional myoelectric control. Journal of Medical Devices 8, 024501.

Huang S, Wensman JP and Ferris DP (2016) Locomotor adaptation by transtibial amputees walking with an experimental powered prosthesis under continuous myoelectric control. IEEE Transactions on Neural Systems and Rehabilitation Engineering 24, 573-581.

Ku PX, Abu Osman NA and Wan Abas WA (2014) Balance control in lower extremity amputees during quiet standing: a systematic review. Gait Posture 39, 672-682.

Ku PX, Osman NA, Yusof A and Abas WW (2012) Biomechanical evaluation of the relationship between postural control and body mass index. Journal of Biomechanics 45, 1638-1642.

Lafond D, Corriveau H and Prince F (2004) Postural control mechanisms during quiet standing in patients with diabetic sensory neuropathy. Diabetes Care 27, 173-178.

Latash ML (2010) Motor synergies and the equilibrium-point hypothesis. Motor Control 14, 294-322.

Lawson BE, Varol HA and Goldfarb M (2011) Standing stability enhancement with an intelligent powered transfemoral prosthesis. IEEE Transactions on Biomedical Engineering 58, 2617-2624.

Legro MW, Reiber GE, Czerniecki JM and Sangeorzan BJ (2001) Recreational activities of lower-limb amputees with prostheses. Journal of Rehabilitation Research and Development 38, 319-325.

Lenzi T, Cempini M, Hargrove L and Kuiken T (2018) Design, development, and testing of a lightweight hybrid robotic knee prosthesis. The International Journal of Robotics Research 37, 953-976.

Liu M, Zhang F, Datseris P and Huang H (2014) Improving finite state impedance control of active transfemoral prostheses using Dempster-Shafer state transition rules. Journal of Intelligent \& Robotic Systems 76, 461-474.

Mansfield A, Danells CJ, Inness E, Mochizuki G and McIlroy WE (2011) Between-limb synchronization for control of standing balance in individuals with stroke. Clinical Biomechanics 26, 312-317.

Mansfield A, Mochizuki G, Inness EL and McIlroy WE (2012) Clinical correlates of between-limb synchronization of standing balance control and falls during inpatient stroke rehabilitation. Neurorehabilitation and Neural Repair 26, 627-635.

Miller JD, Beazer MS and Hahn ME (2013) Myoelectric walking mode classification for transtibial amputees. IEEE Transactions on Biomedical Engineering 60, 2745-2750.

Page DM, George JA, Kluger DT, Duncan C, Wendelken S, Davis T, Hutchinson DT and Clark GA (2018) Motor control and sensory feedback enhance prosthesis embodiment and reduce phantom pain after long-term hand amputation. Frontiers in Human Neuroscience 12, 352.

Quintero D, Villarreal DJ, Lambert DJ, Kapp S and Gregg RD (2018) Continuous-phase control of a powered knee-ankle prosthesis: amputee experiments across speeds and inclines. IEEE Transactions on Robotics 34, 686-701.

Rosenberg DE, Turner AP, Littman AJ, Williams RM, Norvell DC, Hakimi KM and Czerniecki JM (2013) Body mass index patterns following dysvascular lower extremity amputation. Disability and Rehabilitation 35, 1269-1275.

Rouse EJ, Mooney LM, Martinez-Villalpando EC and Herr HM (2013) Clutchable series-elastic actuator: design of a robotic knee prosthesis for minimum energy consumption. IEEE International Conference Rehabilitation Robotics 2013, 6650383.

Rusaw DF and Ramstrand S (2016) Validation of the inverted pendulum model in standing for transtibial prosthesis users. Clinical Biomechanics 31, 100-106. 
Sup F, Varol HA, Mitchell J, Withrow TJ and Goldfarb M (2009) Self-contained powered knee and ankle prosthesis: initial evaluation on a transfemoral amputee. IEEE International Conference Rehabilitation Robotics 2009, 638-644.

Tucker MR, Olivier J, Pagel A, Bleuler H, Bouri M, Lambercy O, Millan Jdel R, Riener R, Vallery H and Gassert R (2015) Control strategies for active lower extremity prosthetics and orthotics: a review. Journal of NeuroEngineering and Rehabilitation $12,1$.

Tucker MR, Shirota C, Lambercy O, Sulzer JS and Gassert R (2017) Design and characterization of an exoskeleton for perturbing the knee during gait. IEEE Transactions on Biomedical Engineering 64, 2331-2343.

Wang J, Kannape O A and Herr HM (2013) Proportional EMG control of ankle plantar flexion in a powered transtibial prosthesis. IEEE International Conference Rehabilitation Robotics 2013, 6650391.

Wen Y, Si J, Brandt A, Xiang G and Huang H (2019 Online reinforcement learning control for the personalization of a robotic knee prosthesis. IEEE Transactions on Cybernetics 50, 2346-2356.

Winter DA, Patla AE, Prince F, Ishac M and Gielo-Perczak K (1998) Stiffness control of balance in quiet standing. Journal of Neurophysiology 80, 1211-1221.

Zhang F and Huang H (2013) Source selection for real-time user intent recognition toward volitional control of artificial legs. IEEE Journal of Biomedical and Health Informatics 17, 907-914.

Ziegler-Graham K, MacKenzie EJ, Ephraim PL, Travison TG and Brookmeyer R (2008) Estimating the prevalence of limb loss in the United States: 2005 to 2050. Archives of Physical Medicine and Rehabilitation 89, 422-429.

Author Biographies

A. Fleming received the BSc degree in Mechanical Engineering from NC State University, in 2013, and he is pursuing a PhD in Biomedical Engineering at the Joint Department of Biomedical Engineering at NC State University and UNC Chapel Hill.

Dr. S. Huang received the BSc degree in Mechanical Engineering from the University of Michigan, in 2005, and also received her MS and $\mathrm{PhD}$ in Biomedical Engineering at the University of Michigan in 2014.

Dr. E. Buxton received her BSc degree in Psychology from the University of North Carolina at Chapel Hill in 2006, and also received her Doctorate of Physical Therapy degree from the University of North Carolina at Chapel Hill in 2009.

F. Hodges received his BA Liberal Arts from Adlerson-Broaddus College. He Founded an Orthotic and Prosthetic Fabrication Facility, SunStone Lab, in 2011 and became a B.O.C. certified Prosthetist Orthotist in 2014.

Dr. H. Huang received the BSc degree from the School of Electronics and Information engineering at Xi'an Jiaotong University in 2000. She received her MS and PhD in Biomedical Engineering at Arizona State University, in 2002 and 2006, respectively.

Cite this article: Fleming A, Huang S, Buxton E, Hodges F and Huang H. H (2021). Direct continuous electromyographic control of a powered prosthetic ankle for improved postural control after guided physical training: A case study. Wearable Technologies, 2, e3, doi:https://doi.org/10.1017/wtc.2021.2 